\title{
La prise en compte de la faune sauvage dans le cadre des procédures d'aménagement, de gestion et d'occupation de l'espace : réalités d'une apparence juridique
}

\author{
Philippe Billet \\ Professeur agrégé de droit public, Le Clos du Bugnon, Grand'Rue 8, 2012 Auvernier, Suisse
}

\author{
Mots-clés : \\ faune sauvage ; \\ aménagement \\ du territoire ; \\ impacts sur \\ l'environnement; \\ étude d'impacts ; \\ analyse \\ «avantage-coût»; \\ compensations
}

\section{Keywords:}

wildlife; area planning; environment impact; impact study; benefit cost analysis; compensation

\begin{abstract}
Résumé - L'occupation de l'espace par l'homme concurrence l'habitat de la faune sauvage, qu'elle détruit, banalise, ou compartimente par des ouvrages et aménagements. Divers instruments ont été développés pour favoriser une coexistence : contrôle de l'occupation des sols dans le respect des intérêts environnementaux, étude d'impact et mesures pour limiter, réduire ou compenser les atteintes, théorie du bilan « coûts-avantages » pour équilibrer préservation de la faune et projets en cause. La concurrence demeure pourtant, du fait de l'utilisation d'outils destinés à gérer les conflits territoriaux humains, inadaptés à la prise en compte de la faune puisque l'étude d'impact et la théorie du bilan " coûts-avantages " n'ont jamais été conçus pour empêcher les aménagements et activités. Il faut donc parfois réduire cette prise en compte de la faune à quelques espaces privilégiés, fragilisés cependant par des dérogations, même strictement encadrées.
\end{abstract}

\begin{abstract}
Taking account of wild fauna within the framework of the activities and procedures of the organization, management and use of grounds legal aspect. The occupation of territory by man poses a threat to wildlife habitats, which may be destroyed, made commonplace or fragmented by the setting-up of unbridgeable or dangerous obstacles. To prevent these injuries, ease them or remedy them, various instruments have been developed to favor a coexistence. The control of the occupation of grounds so as to respect environmental considerations is combined with the balance of the occupation of grounds which defends the law of the town planning, which tends to protect the natural environment. The preliminary study of the incidences on the environment of the projects of development and use of grounds allows to suggest measures for reducing or compensating damage to the environment. The theory of the balance assessment of costs and benefits considers the interests of wildlife conservation as well as those of the projects concerned. The conflict nevertheless remains, since the tools used were originally intended to manage human territorial conflicts, and are not adapted to take account of wildlife. Impact assessments and the theory of the balance assessment were not conceived as tools for the prevention of human activities. It is therefore necessary sometimes to limit allowance for wildlife to a few privileged areas, that may be impaired by certain dispensations even when strictly supervised.
\end{abstract}

Les exploits d'Osmoderma eremita Scopoli, petit scarabée saproxylophage plus connu sous le nom de scarabée Pique prune, capable d'arrêter pendant six ans des bulldozers sur le chantier de l'autoroute A-28 entre Le Mans et Tours sont révélateurs - à la limite de la caricature des enjeux de la prise en compte (ou de l'absence de prise en compte, en l'occurrence) de la faune sauvage dans le cadre des activités et procédures d'aménagement, de gestion et d'utilisation des sols. Contrairement

Auteur correspondant : philippe.billet@free.fr

$\mathrm{Ph}$. Billet est professeur à la faculté de droit de l'université de Bourgogne et président de la Société française pour le Droit de l'environnement (SFDE). à ce qu'un certain battage médiatique a laissé supposer, cependant, occultant au passage d'autres espèces beaucoup plus rares (Blandin et al., 1999 ; Burdeau et al., 2001) ce ne sont pas tant les atteintes ponctuelles, comme ici le défrichement nécessaire au passage d'une voie routière, qui sont à la source de sa disparition, que la gestion des massifs forestiers : les arbres creux dépourvus de valeur commerciale sont éliminés, l'abattage des arbres au milieu de leur espérance de vie prive la forêt de son dernier stade évolutif, la simplification de la structure forestière (remplacement des feuillus par des résineux) prive ainsi cet insecte, et d'autres avec lui, de son biotope que sont les arbres à cavités (Vallauri et al. 2002). 
Il est rare toutefois que les insectes retiennent autant l'attention dans les procédures d'aménagement, leur protection juridique confidentielle expliquant peut-être cela ${ }^{1}$. Les batraciens sont à peine mieux lotis, même si leur sont consacrés des crapauducs et milieux de substitution que sont les mares artificielles, à l'occasion de la construction de voies routières et ferroviaires. Cela illustre cependant que, même à cet échelon du monde animal, l'occupation humaine de l'espace induit le plus souvent un jeu de concurrence avec l'habitat de la faune, qu'elle détruit, banalise, fragmente, barre ou compartimente par l'implantation d'obstacles infranchissables ou dangereux. La dynamique des populations animales implique en effet des déplacements et une répartition sur un territoire donné, que ce soit pour dormir, se nourrir, se reproduire, se protéger ou augmenter son espace vital. Or, tant la zone source, où l'animal accomplit une grande partie de son cycle vital, que les structures qui servent de continuums, peuvent être atteintes et les corridors biologiques affectés par cette occupation humaine (SETRA, 1985, 2000).

Pour prévenir ces déstructurations ou, à défaut, les atténuer, sinon les réparer, divers instruments juridiques ont été développés, tendant à favoriser une certaine coexistence au sein du même espace : le contrôle de l'occupation des sols dans le respect des intérêts environnementaux se conjugue ainsi avec l'équilibre de l'occupation des espaces que défend le droit de l'urbanisme, qui tend à préserver les milieux naturels. L'étude préalable des incidences sur l'environnement des projets d'aménagement et d'utilisation des sols permet de son côté de proposer des mesures afin de limiter, réduire ou, si possible, compenser les atteintes, comme la théorie du bilan " coûts-avantages » tente de faire cohabiter au sein de l'intérêt général l'intérêt de la préservation de la faune et celui des projets en cause. La concurrence demeure cependant, en raison de l'utilisation d'outils destinés à l'origine à gérer les conflits territoriaux humains et réduire les atteintes à l'homme, manifestant leur inadaptation à prendre en compte les intérêts de la faune. Seule cette dernière constituera l'objet de notre étude, compte tenu de son contexte, mais la flore pourrait conduire aux mêmes résultats et peut-être plus encore, compte tenu de la confidentialité dans laquelle elle est tenue, une plante détruite suscitant moins de réactions que la destruction d'un animal ou de son milieu.

\section{La recherche de la coexistence}

De façon assez paradoxale, la prise en compte de la faune dans l'utilisation des sols apparaît dans une

\footnotetext{
1 Arrêté modifié du 22 juillet 1993 relatif à la liste des insectes protégés sur le territoire national, JO 24 septembre 1993, p. 13272.
}

réglementation où la faune est absente : le droit de l'urbanisme. Celui-ci ne vise jamais que l'espace et non les espèces, à travers la recherche d'un équilibre de l'utilisation du territoire. Elle se manifeste, avec plus de vigueur, avec l'anticipation des atteintes qui doit accompagner la plupart des projets d'aménagement.

\section{L'équilibre dans l'utilisation de l'espace}

L'article L. 121-1 du code de l'urbanisme, dans sa dernière rédaction issue de la loi «Solidarité et renouvellement urbains » (SRU) du 13 décembre 2000 - similaire à l'ancienne version de l'article L. 121-10 - impose aux schémas de cohérence territoriale, plans locaux d'urbanisme et autres cartes communales, de déterminer les conditions qui permettent d'assurer l'équilibre entre, d'un côté, le renouvellement urbain, un développement urbain maîtrisé, le développement de l'espace rural et, de l'autre, «la préservation des espaces affectés aux activités agricoles et forestières et la protection des espaces naturels et des paysages, en respectant les objectifs du développement durable». Cette obligation est complétée par celle d'une utilisation économe et équilibrée des espaces naturels et la préservation des écosystèmes, des espaces verts, des milieux, sites et paysages naturels. Cette obligation générale d'équilibre est déclinée dans de nombreuses dispositions du code de l'urbanisme, plus particulièrement pour des milieux fragiles comme la montagne (articles L. et R. 145-1 et suivants) et le littoral (articles L. et R. 146-1 et suivants).

L'équilibre est une notion difficile à définir en ce domaine. Elle a trouvé une place de choix dans la Charte de l'environnement, où elle est évoquée à deux reprises, s'agissant des «équilibres naturels » qui ont conditionné l'émergence de l'humanité, premier considérant de la Charte et comme élément du premier principe consacré, le droit de vivre dans un "environnement équilibré $»^{2}$. La Charte reprend ainsi et complète les "équilibres biologiques » qui doivent être pris en compte dans le cadre de l'analyse des effets directs et indirects, temporaires et permanents d'un projet sur l'environnement imposé par le décret du 12 octobre 1977 sur les études $\mathrm{d}^{\prime}$ impact ${ }^{3}$, ou les milieux littoraux « nécessaires au maintien des équilibres biologiques » protégés par l'article L. 146-6 du code de l'urbanisme. L'équilibre auquel il est fait allusion dans ces textes est un équilibre écologique, instable par nature, le dynamisme d'un écosystème impliquant un jeu permanent entre équilibre et

\footnotetext{
${ }^{2}$ Loi constitutionnelle $n^{\circ} 2005-205,1^{\text {er }}$ mars 2005 relative à la Charte de l'environnement, JO 2 mars 2005 p. 3697.

3 Décret modifié $n^{\circ} 77-1141$ du 12 octobre 1977 pris pour l'application de l'article 2 de la loi n ${ }^{\circ} 76-629$ du 10 juillet 1976 relative à la protection de la nature [Code de l'environnement, art. L 122-1], JO 13 octobre 1977 (désormais art. R. 122-1 s. du code de l'environnement).
} 
déséquilibre, l'évolution d'un équilibre donné vers un nouvel équilibre du fait d'événements, naturels ou non. Il est tout de même surprenant que la notion d'équilibre trouve ainsi sa traduction sans nuance dans le droit à l'heure où elle est quelque peu contestée dans les milieux scientifiques. Le droit semble en faire un idéal d'harmonie, paraît adopter une conception de l'environnement à la Bernardin de Saint-Pierre, largement dépassée pourtant.

L'équilibre prend cependant un tout autre sens lorsque, par le truchement du droit, il met en opposition des intérêts divergents et appelle à la recherche d'une certaine égalité entre ceux-ci, d'une juste proportion entre des éléments opposés. Cette approche de l'équilibre peut s'appréhender à travers la jurisprudence, rare mais significative. Est ainsi illégal le classement de secteurs inventoriés au titre des zones naturelles d'intérêt écologique, faunistique et floristique (ZNIEFF) en zone d'urbanisation future NA, par un plan d'occupation des sols : l'une abrite en effet des espèces rares ou uniques en France où il est recommandé de maintenir le caractère exceptionnel de ce site en y apportant le moins de modifications possibles; l'autre constitue une zone de richesse naturelle unique en Europe, où il est recommandé de maintenir la vocation naturelle des lieux en évitant toute perturbation majeure et notamment les gros aménagements ${ }^{4}$. Viole également ce principe d'équilibre un projet d'unité touristique nouvelle qui prévoit la création de plusieurs golfs et d'un héliport ainsi que la construction de logements, de commerces, d'hôtels, de restaurants pour un total de plus de $60000 \mathrm{~m}^{2}$ de surface hors œuvre nette que l'on se propose d'implanter dans un site naturel d'une qualité exceptionnelle en raison des caractéristiques climatiques qui favorisent, dans la forêt existante, le développement d'une faune variée ${ }^{5}$. Enfin, est illégal un arrêté préfectoral autorisant l'aménagement d'un domaine skiable qui aura un impact très important sur un site sensible « des plus pittoresques du massif des Grandes Rousses » et altérera profondément et de manière irréversible les paysages, la flore et la faune qui comporte des espèces rares, ce projet ne respectant pas la qualité du site dans lequel il s'inscrit et les grands équilibres naturels ${ }^{6}$. Une certaine générale du parti d'urbanisme est donc recherchée, qui associe une mise en perspective avec le reste du document d'urbanisme et le contrôle d'un éventuel déséquilibre induit par le classement de la zone, que ce déséquilibre soit

\footnotetext{
4 Trib. adm. Nice, $1^{\text {er }}$ fév. 1996, Synd. d'exploitants agricoles de Fontau-Sorge et autres, Études foncières, décembre 1996, p. 40, note Lamorlette.

5 Trib. adm. Grenoble, 10 mai 1990, Ass. «Club alpin français », Revue juridique de l'environnement 1991, p. 225.

${ }^{6}$ Cons. d'État, 4 août 1994, Commune de Vaujany, requête $n^{\circ}$ 129898. Dans le même sens, à propos du littoral, Cour administrative d'appel Marseille, 25 novembre 2004, Commune de La Croix Valmer, requête n 99MA01948.
}

créé ou accentué par celui-ci (Chauvin, 1996). La faune n'est cependant prise en compte qu'incidemment, dans la mesure où seul son territoire est concerné : les effets différés de la planification et des aménagements qu'elle autorise sur la quiétude des espèces, la pérennité de leur zone d'alimentation et de reproduction ne sont jamais envisagés en tant que tels.

Les dispositions d'ordre public du règlement national d'urbanisme qui s'appliquent, que la commune soit dotée ou non d'un plan local d'urbanisme approuvé ou document en tenant lieu, ne compensent que partiellement ce déséquilibre : elles ne concernent en effet que le projet et non la zone qui en constitue l'assiette, d'une part et, d'autre part, leur champ d'application ne vise que les projets soumis à permis de construire, ce qui exclut de nombreux aménagements comme les routes et les voies ferrées. Le permis de construire doit ainsi être délivré « dans le respect des préoccupations d'environnement définies à l'article $1^{\text {er }}$ de la loi du 10 juillet 1976 relative à la protection de la nature. Il peut n'être accordé que sous réserve de l'observation de prescriptions spéciales, si les constructions, par leur situation, leur destination ou leurs dimensions, sont de nature à avoir des conséquences dommageables pour l'environnement ». Cependant, la lettre même du texte interdit de refuser le permis de construire ${ }^{7}$ : tout au plus peut-il être annulé pour erreur manifeste d'appréciation si les prescriptions destinées à prendre en compte la faune sont insuffisantes $^{8}$. Les juridictions administratives font, en outre, une interprétation tendancieuse de cette disposition : elles n'en admettent l'application que pour préserver des espèces animales et végétales inscrites sur la liste des espèces protégées, alors que le texte ne prévoit pas une telle limitation ${ }^{9}$. Par ailleurs, ces mêmes juridictions admettent l'absence de toute mesure de protection dès lors que l'aménagement en cause n'est pas incompatible avec les règles de protection des espèces concernées. Elles se contentent alors de la prescription de mesures de remise en état des lieux destinées à atténuer les atteintes : «les dispositions envisagées pour la remise en état du site après la réalisation de l'ouvrage devraient limiter son impact sur l'environnement. » "L'impact de la réalisation de cet ouvrage sur l'environnement sera

\footnotetext{
7 Cour adm. appel Lyon, 6 juin 2000, Commune de Hyèresles-Palmiers, requête n ${ }^{\circ}$ 95LY00062 -Cons. d'État, 7 février 2003, Sté civile d'exploitation agricole Le haras d'Ardères II, requête $\mathrm{n}^{\circ} 220215$.

8 Trib. adm. Lille, 12 janvier 1995, Assoc. Ardelot, Opale, Environnement, Études foncières n ${ }^{\circ} 66$, mars 1995, p. 54 - Cons. d'État, 15 janvier 1999, Sté Omya, Gazette du Palais 29-30 décembre 1999, p. 30, solution a contrario-Cour adm. appel Nancy, 23 mars 1995, Assoc. Haute Tension en Charolais-Brionnais, requête $\mathrm{n}^{\circ} 93 \mathrm{NC} 00715$ sol. a contrario.

9 Cour adm. appel Marseille, 2 mars 2000, Comité de défense de Vingrau et M. Bassou, requête n 97MA10087 s.
} 
limité et que les dispositions prévues pour la remise en état des lieux en atténueront les effets négatifs ${ }^{10}$. Autrement dit, l'existence d'effets négatifs est reconnue mais les atteintes actuelles sont admises parce que limitées, sans pour autant faire disparaître ces effets. L'autorisation d'édifier l'ouvrage est cependant validée parce que, lorsque l'ouvrage cessera de fonctionner (à une date non précisée), les lieux seront remis en l'état. Les effets actuels sont ainsi à peine pris en considération, du moment qu'ils restent compatibles avec la protection recherchée. Or, à la différence de la conformité, rétive à toute adaptation, la compatibilité offre une marge d'appréciation certaine, difficilement admissible cependant s'agissant de la protection de milieux fragiles : il est simplement recherché une absence de contrariété, qui n'interdit pas de ce fait les atteintes, du moment qu'elles restent limitées. Le dispositif de l'article R. 111-14-2 ne perd cependant pas de son intérêt dans la mesure où il peut amener le pétitionnaire à reconsidérer son projet, le cas échéant en s'appuyant sur les résultats d'une étude d'impact, pour tenir compte des préoccupations des protections de la faune et, plus généralement, de l'environnement ${ }^{11}$. Il permet en tout cas de vraiment anticiper les atteintes à la faune, de façon plus ponctuelle sans doute que les documents d'urbanisme, mais surtout plus efficiente dans la mesure où ce n'est plus le zonage et l'occupation des sols rendue possible qui doivent être accompagnés de mesures de réduction ou de compensation, mais le projet lui-même.

L'incapacité du droit de l'urbanisme à traiter du problème de la faune sauvage est flagrante, mais elle n'est que le reflet d'un conditionnement de la norme : il n'a tout simplement pas été conçu pour cela. Cette inadéquation trouve sa traduction dans le principe selon lequel « les préoccupations d'environnement sont prises en compte par les documents d'urbanisme dans le cadre des procédures qui leur sont propres ", ainsi que le prévoit l'article L 122-1 du code de l'environnement. Cette exigence s'est traduite par l'obligation, pour certains documents d'urbanisme, de comporter une étude d'environnement, dont il a été souligné qu'elle n'était en rien une étude $\mathrm{d}^{\prime}$ impact $^{12}$. En dépit de toilettages textuels, cette étude est restée très fruste, limitée à une analyse de l'état initial de l'environnement et à celle des incidences prévisibles de sa mise en œuvre sur l'environnement, suivie d'un exposé de la manière dont ce document prend en compte le souci de sa préservation et de

\footnotetext{
10 Ainsi, à propos d'un barrage : Cons. d'État, 14 avril 1999, Comité de sauvegarde de la Vallée de Chambonchard et autre, requête $n^{\circ} 193363$; Cour adm. appel Marseille, 18 octobre 2001, Assoc. nationale de protection des salmonidés «Truite, ombre, saumon » TOS, requête $n^{\circ}$ 98MA00194.

11 Cons. d'État, 9 octobre 1993, Commune de Grigny, requête $\mathrm{n}^{\circ} 101681$.

12 Cons. d'État, 24 juillet 1981, Assoc. pour la sauvegarde du pays de Rhuys, JCP G 1982, II, 19798, conclusions B. Genevois.
}

sa mise en valeur. On aurait pu espérer une évolution avec l'évaluation environnementale qui doit désormais accompagner l'adoption de certains documents d'urbanisme, ainsi que l'a imposé une ordonnance du 3 juin $2004^{13}$. Cependant, le décret du 27 mai $2005^{14}$ qui la met en œuvre réduit quelque peu les espérances que l'on pouvait mettre en elle : si le contenu du rapport de présentation des documents d'urbanisme en sort renforcé en ce qui concerne la prise en compte de l'environnement, c'est d'un environnement spatial dont il s'agit principalement. Doivent en effet y figurer l'analyse des caractéristiques des zones susceptibles d'être touchées de manière notable par la mise en œuvre du document, les motifs de la délimitation des zones. Le biotope n'est pas identifié en tant que tel : ce n'est qu'incidemment qu'on le découvre, avec l'exposé des problèmes posés par l'adoption du document d'urbanisme sur la protection des zones revêtant une importance particulière (tels les sites Natura 2000). La faune n'est également jamais mentionnée et il faut faire preuve d'un certain optimisme pour imaginer qu'elle ne sera pas négligée par «l'analyse des incidences notables prévisibles de la mise en œuvre du document sur l'environnement » que doit comporter cette évaluation environnementale (Billet, 2005). Le législateur comme le pouvoir réglementaire semblent être passés à côté d'une bonne occasion pour « racheter » le droit de l'urbanisme de ses errements, n'ayant pas osé faire de cette évaluation une véritable étude d'impact, afin de vraiment anticiper ces atteintes et les corriger le plus tôt possible.

\section{L'anticipation des atteintes}

L'anticipation des atteintes à la faune apparaît en effet comme le meilleur remède à celles-ci. C'est la voie qui a été consacrée par l'étude d'impact issue de la loi du 10 juillet 1976 relative à la protection de la nature $^{15}$ et par l'étude des incidences des projets publics et privés sur l'environnement issue de la directive communautaire du 27 juin $1985^{16}$, ainsi que leurs diverses

\footnotetext{
13 Ord. $\mathrm{n}^{\circ}$ 2004-489, 3 juin 2004, JO, 5 juin 2004, p. 9979 et rectificatif $J O, 10$ juillet 2004, p. 12554. Code de l'environnement, article L. 122-4 et code de l'urbanisme, article L. 121-15.

14 Décret n ${ }^{\circ}$ 2005-608 du 27 mai 2005 relatif à l'évaluation des incidences des documents d'urbanisme sur l'environnement et modifiant le code de l'urbanisme, JO, 29 mai 2005, p. 9499. Code de 1'urbanisme, articles R. $122-2,5^{\circ}$ et R. $123-2-1,4^{\circ}$.

15 Code de l'environnement, article L. 122-1.

16 Directive $n^{\circ}$ 85/337/CE du Conseil du 27 juin 1985 relative à l'évaluation des incidences de certains projets publics et privés sur l'environnement, JOCE $\mathrm{n}^{\circ}$ L. 175, 5 juillet 1985, modifiée en dernier lieu par Directive $n^{\circ}$ 2003/35/CE du Parlement européen et du Conseil du 26 mai 2003, JOCE n L. 156, 25 juin 2003.
} 
déclinaisons ${ }^{17}$. Cette anticipation n'a cependant de sens qu'accompagnée des diverses mesures d'évitement des atteintes que ses conclusions permettent d'envisager. Ainsi, l'étude d'impact impose, dans sa configuration actuelle, de réaliser une analyse de l'état initial du site et de son environnement portant notamment sur les richesses naturelles et les espaces naturels, une analyse des effets directs et indirects, temporaires et permanents $\mathrm{du}$ projet sur l'environnement, et en particulier sur la faune, les milieux naturels et les équilibres biologiques et de présenter les mesures envisagées par le maître de l'ouvrage ou le pétitionnaire pour supprimer, réduire et, si possible, compenser les conséquences dommageables du projet sur l'environnement, ainsi que l'estimation des dépenses correspondantes ${ }^{18}$.

Un tel dispositif implique donc au préalable d'appréhender correctement la richesse et la variété de la faune sauvage présente sur le secteur concerné par le projet, dès lors que cela conditionne les mesures à adopter en sa faveur. Cette présence peut être avérée, mais également fortement vraisemblable, compte tenu de la présence de l'habitat potentiel de telle ou telle espèce non formellement rencontrée. A donc été jugée illégale une autorisation reposant sur une étude d'impact qui ne faisait pas état de la présence d'une espèce, qu'elle soit ou non protégée $^{19}$, ou qui ne donnait aucune précision sur son importance, sa répartition et sur les conséquences possibles de l'autorisation litigieuse sur son devenir ${ }^{20}$. Si le juge admet des omissions, à condition qu'elles ne soient pas déterminantes ${ }^{21}$, il sanctionne les autorisations de projets dont les atteintes auraient été mal - ou pas du

17 Comme l'étude des incidences sur l'eau et les milieux aquatiques de l'article 2 du décret modifié n 93-742 du 29 mars 1993 relatif aux procédures d'autorisation et de déclaration prévues par les articles L. 214-1 et suivants du code de l'environnement: JO 30 mars 1993. Cette étude impose d'analyser les incidences de l'opération envisagée notamment sur le milieu aquatique et la vie biologique, et de préciser les mesures compensatoires ou correctrices envisagées.

18 Code de l'environnement, art. R. 122-3, 4.

${ }^{19}$ Cons. d'État, 9 décembre 1988, Entreprise de dragage et de travaux publics et Sté d'exploitation de la Garonne, Revue juridique de l'environnement, 1989/2, p. 187 - Trib. adm. Pau, 19 décembre 2002, SEPANSO Béarn Pyrénées, Revue juridique de l'environnement 2004/1, p. 70, note J. Sironneau - Trib. adm. Clermont-Ferrand, 24 juin 2003, Assoc. Collectif SaintVictor-sur-Arlanc c/ Préfet Haute-Loire : JCP AdministrationsCollectivités territoriales 2003, $\mathrm{n}^{\circ}$ 1137, note Ph. Billet.

20 Trib. adm. Amiens, 20 novembre 1992, Assoc. AisneEnvironnement, Revue juridique de l'environnement 1993/4, p. 577.

21 « $\mathrm{Si}$, en ce qui concerne la faune et la flore, quelques espèces observées... ont été omises dans l'étude, cela est sans incidence sur la qualité de l'information apportée au public et à ceux qui ont à en connaître » (Cons. d'État, 4 juillet 1994, SA Ets CatteauLanglois et autres, requête $n^{\circ} 119303$ s.) - «Si quelques inexactitudes et lacunes ont pu être relevées, l'analyse de l'état initial du site ne comporte pas d'erreurs ou d'omissions importantes » tout - évaluées, ou mal - ou pas du tout - prévenues ou compensées. De fait, si l'étude d'impact d'un projet a bien appréhendé la richesse et la variété de la faune sauvage présente sur le secteur concerné par le projet, l'autorisation de le réaliser est illégale dès lors qu'elle ne présente pas les mesures de protection de l'environnement exigées $^{22}$. La plupart du temps, cependant, les requérants omettent d'assortir leur recours d'une demande de suspension d'exécution, pourtant facilitée par 1'article L. 1222 du code de l'environnement. Il en résulte que lorsque le juge de première instance se prononce sur le fond, et plus encore le Conseil d'État, le projet est souvent achevé depuis longtemps et l'ouvrage a été mis en service. Le régime de droit commun du référé suspension impose, on le sait, que le requérant démontre l'urgence de la situation et rapporte l'existence d'un doute sérieux quant à la légalité de la décision, le juge disposant par ailleurs d'un pouvoir discrétionnaire pour prononcer cette suspension si les deux conditions requises sont vérifiées ${ }^{23}$. Le code de l'environnement va au-delà en prévoyant que si une requête déposée devant la juridiction administrative contre une autorisation ou une décision d'approbation d'un projet soumis à étude d'impact est fondée sur l'absence de cette étude, la juridiction saisie doit faire droit à la demande de suspension de la décision attaquée, dès que cette absence est constatée par une procédure d'urgence $^{24}$. Il est sans doute nécessaire de rapporter une absence d'étude d'impact, mais les juridictions administratives admettent que cette condition est vérifiée lorsque l'étude d'impact, bien que présente sous cette dénomination dans le dossier, est affectée d'une insuffisance manifeste, flagrante, équivalente à une absence, notamment pour ne pas avoir pris en compte un élément substantiel. L'intérêt d'un tel référé, outre la rapidité d'intervention du juge $\mathrm{e}^{25}$, est que si l'ordonnance du juge des référés n'a pas l'autorité de la chose jugée, elle est néanmoins exécutoire et obligatoire, en vertu de l'autorité qui s'attache aux décisions de justice : lorsque sa décision a été suspendue, l'autorité administrative ne peut donc plus prendre de nouveau la même décision. Elle ne peut donc pas contrarier les effets de l'ordonnance de référé en l'annihilant par une nouvelle décision identique à celle qui a

(Cons. d'État, 14 avril 1999, Commune de La Petite Marche, requête $n^{\circ}$ 185935).

22 Cons. d'État, 11 mars 1996, Commune d'Orelle, requête $\mathrm{n}^{\circ} 140275$.

${ }^{23}$ Code de justice administrative, article L. 521-1. Voir B. Busson (2001: 93).

24 Cons. d'État, 14 mars 2001, Commune de Goutrens, Revue française de droit administratif 2001, p. 762, conclusions Chauvaux.

${ }^{25}$ Généralement entre 20 et 40 jours entre le jour de la saisine et le prononcé de la décision avant-dire droit, alors qu'il faut entre 9 et 14 mois pour qu'il se prononce sur le fond en première instance. 
été suspendue $\mathrm{e}^{26}$. Un autre intérêt de cette suspension est d'éviter les annulations tardives d'autorisation d'un ouvrage déjà achevé et les conséquences de la qualification d'ouvrage public de cet ouvrage : à la suite d'une lente évolution jurisprudentielle, le juge administratif peut désormais, en fonction des conclusions du propriétaire requérant ou de ses ayants droit, enjoindre sous astreinte la personne qui a édifié l'ouvrage de remettre les lieux en état, dès lors que la démolition de l'ouvrage constitue la conséquence nécessaire du respect de la chose jugée. Cependant, « il lui appartient, pour déterminer en fonction de la situation de droit et de fait existant à la date à laquelle il statue, si l'exécution de cette décision implique qu'il ordonne la démolition de cet ouvrage, de rechercher d'abord si, eu égard notamment aux motifs de la décision, une régularisation appropriée est possible; que, dans la négative, il lui revient ensuite de prendre en considération d'une part les inconvénients que la présence de l'ouvrage entraîne pour les divers intérêts publics ou privés en présence et notamment, le cas échéant, pour le propriétaire du terrain d'assiette de l'ouvrage, d'autre part les conséquences de la démolition pour l'intérêt général, et d'apprécier en rapprochant ces éléments, si la démolition n'entraîne pas une atteinte excessive à l'intérêt général ${ }^{27}$. Autrement dit, même si le projet est illégal, y compris pour des raisons tenant à une insuffisance de l'étude d'impact au regard des intérêts faunistiques, le juge peut, à la suite de l'annulation de l'autorisation relative au projet, refuser d'ordonner la démolition de l'ouvrage pour des raisons d'intérêt public.

En tout état de cause, l'existence de l'étude d'impact, sa philosophie même, n'ont pas pour vocation de ruiner un projet mais d'améliorer son intégration au regard des conditions environnementales. L'étude d'impact n'aboutit jamais à remettre en cause un projet dans son principe, mais à l'améliorer dans sa configuration. Elle tend à atténuer les antagonismes entre aménagement et faune, mais sans jamais pouvoir totalement les effacer, en raison de l'artificialisation du milieu liée à la réalisation de l'aménagement.

\section{La consécration des antagonismes}

L'implantation d'un aménagement, quel qu'il soit, dans un milieu donné, porte indéniablement atteinte à ce milieu et affecte directement ou indirectement la faune

\footnotetext{
${ }^{26}$ Cons. d'État, Section, 5 novembre 2003, Convention vie et nature pour une écologie radicale et Association pour la protection des animaux sauvages, Actualité juridique droit administratif 2003, p. 2253, chron. Donnat et Casas.

27 Cons. d'État, Section, 29 janvier 2003, Syndicat départemental de l'électricité et du gaz des Alpes-Maritimes et Cne de Clans, Revue française de droit administratif 2003/3, p. 477, conclusions Maugüé et note Lavialle.
}

qui y vit ou le fréquente. Diverses procédures, présentées ordinairement comme permettant d'intégrer l'environnement dans le processus décisionnel, apparaissent à l'analyse comme des alibis justifiant la réalisation dudit projet. Une lecture un peu différente de la théorie du bilan « coûts-avantages » permet de s'en assurer, de même que la compensation des atteintes.

\section{La théorie du bilan, justification des déséquilibres}

Lorsque, en 1971, le Conseil d'État a inventé la théorie du bilan "coûts-avantages » dans son arrêt «Ville Nouvelle-Est », il ne s'agissait pas tant pour lui de définir une nouvelle méthode d'analyse pour contrôler la légalité d'un projet d'aménagement que de permettre, par une comparaison des avantages et des inconvénients d'un projet, de justifier celui-ci. Ainsi, selon une formule devenue classique, «[...] une opération ne peut être légalement déclarée d'utilité publique que si les atteintes à la propriété privée, le coût financier et éventuellement les inconvénients d'ordre social qu'elle comporte ne sont pas excessifs eu égard à l'intérêt qu'elle présente. ${ }^{28}$. Certes, traditionnellement, le juge administratif vérifiait si l'opération présentait en elle-même une utilité publique, in abstracto, sans s'attacher à ses inconvénients, affaiblissant ainsi son contrôle puisqu'il admettait l'utilité publique de toute opération présentant un caractère d'intérêt général. La théorie du bilan lui permet donc de renforcer ce contrôle. Cependant, si les atteintes à l'environnement ont, par la suite, complété le plateau des «inconvénients » ${ }^{29}$ la pesée globale des intérêts propre à ce mode de contrôle de légalité opère un amalgame : devant statuer sur une opération dont l'utilité publique est contestée, le juge administratif admet que les effets négatifs de celle-ci, notamment son impact écologique, sont gommés par les avantages qu'elle présente. Or la jurisprudence révèle qu'il l'admet presque toujours au bénéfice de l'habitation, de l'emploi, des grands aménagements du territoire ou de la circulation automobile, au terme d'une démarche "qui évoque le truquage » (Holleaux, 1981), une balance qui pencherait toujours du même côté. Il opère en quelque sorte une hiérarchisation des valeurs : ainsi, à propos d'une voie routière, "les inconvénients que présente le tracé retenu par le décret attaqué et qui tiennent notamment aux atteintes portées à l'environnement, aux risques de perturbation des déplacements de la faune... ne sont pas excessifs eu égard à

\footnotetext{
${ }^{28}$ Cons. d'État, Ass., 28 mai 1971, Min. équipement et du logement c/ Fédération de défense des personnes concernées par le projet actuellement dénommé «Ville nouvelle Est »: Rec. CE, p. 409, conclusions Braibant; in Long, M., et al., 2005, Grands arrêts de la jurisprudence administrative, Paris, Dalloz.

29 Cons. d'État, 25 juillet 1975, Syndicat CFDT des marinspêcheurs de la rade de Brest : Rec. CE, tables, p. 1148.
} 
l'intérêt que revêt la construction de la déviation » ${ }^{30}$. Il n'y a pas d'exemples, dans la jurisprudence, où le juge retient les seules atteintes à la faune comme justifiant l'annulation de la déclaration d'utilité publique d'un projet ${ }^{31}$. Son approche est beaucoup plus globale et la faune est réduite à être un élément parmi d'autres du bilan. Ainsi, l'annulation de la déclaration d'utilité publique d'un barrageréservoir sur la Trézence (Charente-Maritime) doit moins «à la forte charge de cette eau en matière organique serait susceptible d'avoir des conséquences négatives sur les poissons vivant dans les cours d'eau situés en aval ainsi que sur les huîtres et les autres coquillages du bassin de Marennes-Oléron » qu'au coût du projet ${ }^{32}$. Comme a pu le noter Frédéric Rouvillois (1997), cette jurisprudence consacre un «pseudo-bilan », tant la pesée caractéristique du contrôle du bilan apparaît superflue : «C'est parce que l'intérêt apparaît incertain que le coût est mis en avant, comme le moyen le plus simple de montrer que la "balance" penche en défaveur de l'opération. » Dans cette hypothèse, cette prise en considération révèle «le caractère fictif d'un tel contrôle ». En définitive, peu importe le coût du projet : le seul fait que ce coût existe suffit à le rendre excessif, "sans qu'il soit nécessaire d'ajouter quoi que ce soit au passif de l'opération ", conclut-il. Exit l'intérêt faunistique, d'autant plus que, dans cette affaire, le Conseil d'État relevait que « les avantages que le barrage pourrait présenter pour l'agriculture ne figurent pas parmi les objectifs de l'opération ", indiquant à mimots que si de tels avantages avaient été mis en avant, le projet aurait éventuellement pu être déclaré d'utilité publique. La faune ne pèse donc pas bien lourd face aux aménagements. Il ne faut toutefois pas nier les effets préventifs de ce contrôle : la crainte des effets d'un recours incite souvent les aménageurs à se ranger aux exigences écologiques et à soumettre leur projet à des études scientifiques parfois très poussées, voire à s'adjoindre les services d'associations de protection de l'environnement qui connaissent bien le secteur pour réaliser les études. Il en va de même lorsque les projets de déclarations d'utilité publique prononcées par décret font l'objet d'un examen

\footnotetext{
30 Cons. d'État, 7 décembre 1979, Association fédérative régionale pour la protection de la nature, requête $\mathrm{n}^{\circ} 11081$.

31 Les atteintes à l'environnement ne sont retenues comme invalidant le projet qu'en raison de leur gravité au regard de la sensibilité du milieu : ainsi, à propos d'une opération touristique dans un site inscrit (Cons. d'État, 26 mars 1980, Premier ministre c/ Vve Beau de Loménie : Rec. CE, p. 171). En revanche, sa protection est retenue comme justifiant l'utilité publique de l'opération lorsqu'il s'agit d'une acquisition en vue de le protéger (Cons. d'État, 12 avril 1995, Conservatoire de l'espace littoral et des rivages lacustres : Rec. CE, p. 162 ; Actualité juridique droit administratif 1995, p. 660, note Hostiou).

32 Cons. d'État, 29 octobre 2003, Ass. SOS-Rivières et environnement et autres, JCP Administrations-Collectivités territoriales 2003, $\mathrm{n}^{\circ}$ 2108, note Ph. Billet; Revue juridique de l'environnement 2004/2, p. 210, note J. Sironneau.
}

préalable par la Section des travaux publics du Conseil d'État.

Cette constitution d'une telle échelle des valeurs est suspecte par essence, mais il paraît difficile de reprocher au droit de se faire le reflet d'une réalité tout aussi contestable qui affecte la réalisation des études environnementales. L'évaluation engage en effet l'évaluant, qui engage lui-même ses propres valeurs : un spécialiste de tel ou tel milieu ou de telle ou telle espèce aura beaucoup plus d'inclinaison à défendre ce qu'il connaît bien, mû par l'intérêt qu'il y porte, plutôt que de mettre en avant ce à quoi il ne porte qu'un relatif intérêt ou dont il n'a qu'une connaissance approximative (ceci expliquant peut-être cela). Il va donc porter le résultat de son étude et rechercher un consensus autour des valeurs qu'il défend, orientant ainsi les choix de l'aménageur, à moins qu'il ne soit porté par les siens qui correspondent à sa propre échelle de valeurs. Il est extrêmement rare au demeurant qu'un bureau d'étude, choisi pour réaliser une étude d'impact ou toute autre étude d'environnement, conclut à la nécessité d'abandonner le projet au titre des alternatives qui s'offrent au maître d'ouvrage. Une approche plus consensuelle va influencer le résultat de l'évaluation, consacrant les intérêts bien compris de l'un et de l'autre. Le référentiel est alors faussé par des échelles de valeurs qui ne relèvent pas toujours de la rationalité scientifique. On voit alors mal comment le juge administratif peut trancher lorsqu'il est saisi de la contestation d'un projet au motif de l'insuffisance du contenu de l'étude d'impact. Il est naturellement peu enclin à diligenter des contre-expertises pour apprécier la réalité d'une erreur manifeste d'appréciation - qui auront de toutes façons les mêmes défauts et si l'erreur est «manifeste", point n'est besoin d'une telle contre-expertise - et tout aussi peu enclin à admettre les démonstrations de requérants dans un débat qu'il considère souvent comme étant idéologique. Il saura en revanche se montrer beaucoup plus sensible aux compensations offertes par le maître d'ouvrage qui, de ce fait, permettront de faire pencher la balance en faveur du projet.

\section{La compensation, alibi de l'aménagement}

La compensation est devenue un credo de la politique d'aménagement, à un point tel qu'elle en a occulté les deux autres dispositifs prévus par le régime des études d'impact que sont la limitation et la réduction des impacts. Solution de "facilité » s'il en est, la compensation permet de ne pas avoir à rechercher des solutions d'évitement des espaces fréquentés par la faune sauvage. Jean Untermaier (1986) n'a d'ailleurs pas hésité à la qualifier de "supercherie », en estimant que la compensation n'a véritablement de sens «qu'à la condition de remplacer ce qui a disparu par autre chose, non seulement de même valeur, mais aussi de même nature ». Ainsi, « La 
compensation d'un impact négatif sur le milieu naturel ne peut être qu'écologique. Il arrive pourtant qu'elle revête la forme de mesures financières ou techniques, ce qui est une aberration et, à l'occasion, aggrave le préjudice. Compenser par exemple, la destruction, ici, d'un étang par la création, là, d'une base de loisirs, constitue une application dévoyée de la loi du 10 juillet 1976 ». Si l'on rajoute que, dans le cadre d'une étude d'impact, les effets environnementaux de la mesure compensatoire ne sont eux-mêmes pas analysés, on ne peut que se montrer sceptique quant aux effets protecteurs de telles mesures.

On ne sait d'ailleurs le plus souvent pas grand-chose de ces mesures, que les juridictions administratives se contentent de citer comme un standard : ainsi, à propos de la mise à deux fois deux voies d'une route nationale " en ce qui concerne la faune et la flore, cette étude prend en compte l'ensemble des données disponibles sur les espèces les plus remarquables et présente des propositions de mesures compensatoires $»^{33}$. Dans certains cas cependant, plus rares, les mesures font l'objet de descriptions précises. Ainsi, à propos d'un projet d'exploitation de carrière : «s'agissant de l'aigle de Bonelli, l'objet même des bâtiments est de réduire les bruits consécutifs à l'exploitation de l'installation de broyage, concassage, criblage, cette réduction étant obtenue par un bardage en matériaux à isolation phonique renforcée; qu'en outre, la société s'est engagée à mettre en œuvre les mesures compensatoires proposées par les associations du Grive et du Gor, associations régionales coordonnant la protection de l'aigle de Bonelli, notamment la réalisation de cultures cynégétiques appropriées et la création de points d'eau » ${ }^{34}$. De même, s'agissant de l'aménagement $\mathrm{d}^{\prime}$ une route nationale, la réalisation de "passages pour la faune, et banquette enherbée pour tous les ouvrages hydrauliques, submersible en période de hautes eaux, destinée à la petite faune » permet de réduire l'effet de coupure ${ }^{35}$.

L'analyse des effets de ces mesures comme compensations effectives n'est cependant jamais abordée, même s'il est parfois prévu un suivi de l'espèce sur quelques années et d'évaluation des mesures adoptées. L'efficacité relative des crapauducs (ou batrachoducs) ${ }^{36}$ comme celle des tunnels pour assurer le passage des mammifères (SETRA, 1993, 2000) remet en cause la transparence des infrastructures linéaires et marque, dans une certaine mesure, l'échec de la compensation, alors même qu'elle

\footnotetext{
${ }^{33}$ Cons. d'État, 9 février 2004, Ass. Manche Nature, requête $\mathrm{n}^{\circ} 223121$.

34 Cons. d'État, 15 janvier 1999, Sté Omya, préc. note 8.

35 Cons. d'État, 25 juin 2003, Commune de Dampvalley-lesColombes, requête $\mathrm{n}^{\circ} 244712$.

${ }^{36}$ Dont les résultats sont présentés comme «variables, qui ne dépendent pas toujours du soin pris à leur mise en place» (Duguet et Melki, 2003), ou dont la conception peut ne pas toujours être adaptée à une espèce en particulier (Lesbarrères, 2003).
}

aura permis de valider le projet. On en voudra également pour exemple le suivi de certaines espèces de l'avifaune pendant quelques années, comme cela a été prévu dans l'étude d'impact, puis qui font l'objet de prédation après l'achèvement de la période de suivi, faute de surveillance.

Une des applications les plus préjudiciables du mécanisme de la compensation reste celle qui en est faite dans le cadre des aménagements susceptibles d'affecter les sites Natura 2000. L'article L. 414-4 du code de l'environnement prévoit ainsi que lorsqu'il n'existe pas d'autre solution que la réalisation d'un programme ou projet qui est de nature à porter atteinte à l'état de conservation du site, «l'autorité compétente peut donner son accord pour des raisons impératives d'intérêt public ». "Dans ce cas, elle s'assure que des mesures compensatoires sont prises pour maintenir la cohérence globale du réseau Natura 2000. Ces mesures compensatoires sont à la charge du bénéficiaire des travaux, de l'ouvrage ou de l'aménagement ». Or, ces mesures ne sont destinées qu'à permettre le maintien de la cohérence globale du réseau Natura 2000. Du milieu atteint et de la faune qui le colonise, point n'est question : ils sont purement et simplement sacrifiés sur l'autel de l'aménagement au prétexte du rétablissement de cette cohérence par la sauvegarde d'un autre milieu. «Compenser et détruire», résumait sobrement Jean Untermaier (1986), après avoir qualifié la compensation de "supercherie». Sans doute un début de réponse peut-il être trouvé dans l'étude d'impact qui doit accompagner le projet, mais on conçoit sans peine que la compensation des atteintes analysée dans cette étude d'impact parallèle se référera aux mesures compensatoires "prises pour maintenir la cohérence globale du réseau Natura $2000 »$. Cercle vicieux, finalement, dont seul le projet d'aménagement sortira finalement vainqueur, car on voit mal l'État sanctionné par la Cour de justice des Communautés européennes être condamné à remettre le site désormais aménagé dans son état antérieur, solution qui s'avère écologiquement impossible ou presque. On peut du reste se demander si le recours à cette notion de « cohérence » est bien fondé scientifiquement, la référence à son appréhension «globale » laissant en tout cas supposer qu'elle peut admettre çà et là quelques atteintes à l’homogénéité qu'elle appelle.

Cet échange «de bons procédés » n'est en tout cas pas si nouveau, si l'on se souvient de l'épisode du projet d'extension du domaine skiable des Grands-Montets, dans le site classé du mont Blanc : il s'agissait en l'occurrence d'autoriser l'aménagement de la montagne de Lognan et la combe de la Pendant pour créer un nouveau centre d'animation desservi par télésiège et de quatre remontées mécaniques dans le site classé du mont Blanc. La contrepartie était une révision du plan d'occupation des sols de la commune de Chamonix dans le sens d'une réduction de l'urbanisation, le site de Carlaveyron devant par ailleurs faire l'objet d'une procédure de classement, pour 
partie au titre de la loi du 2 mai 1930 sur les monuments naturels et les sites, pour partie en tant que réserve naturelle (Untermaier, 1986). Ce précédent a fait florès, si l'on en juge par le déclassement de la réserve naturelle de l'Iseran, motivé pour une large part par le souhait des élus et aménageurs de pouvoir étendre par la station de Val d'Isère le domaine skiable du col de l'Iseran, les 1500 hectares déclassés étant compensés par la création de la réserve naturelle de Bailletaz sur 495,23 hectares, outre la prescription d'arrêtés de biotopes sur le territoire de l'exréserve de l'Iseran et la création d'une réserve de chasse sur le glacier du Pissaillas ${ }^{37}$. Le dernier épisode en date de l'arrêté du 30 mai 2005 portant création d'une zone de protection de biotope en vue du déclassement partiel de la réserve naturelle de la baie de la Canche ${ }^{38}$ décrédibilise plus encore la compensation telle qu'elle est conçue par l'autorité administrative.

À côté de la compensation, tend à se développer une autre mesure d'anticipation des atteintes, entérinée par les juridictions administratives : le déplacement des espèces à des fins de sauvegarde ${ }^{39}$. Si elle est justifiable, en ce sens qu'elle évite effectivement les atteintes par l'aménagement lui-même, elle n'est pas toujours couronnée de succès sur le long terme et marque surtout l'échec de l'intégration environnementale du projet - qui se résume finalement à une intégration paysagère -, soulignant un peu plus l'antagonisme entre l'aménagement en cause et le milieu dans lequel il s'inscrit, avec lequel il ne peut pas composer. Étude d'impact et théorie du bilan n'ont jamais été conçues, loin s'en faut, comme des outils destinés à empêcher les aménagements et activités humaines mais, souvent, comme des justifications de ceux-ci. Il faut donc parfois réduire cette prise en compte de la faune à quelques espaces privilégiés, fragilisés cependant par des dérogations qui restent toujours possibles, même strictement encadrées.

Cette situation résume à elle seule toute l'ambiguité des mécanismes de prévention des atteintes à la faune à l'occasion des projets d'aménagements : quelle que soit sa dénomination, la mesure préventive, compensatoire ou réparatrice n'apparaît en définitive que comme la contrepartie de la destruction du milieu naturel, justifiant la réalisation du projet sans jamais véritablement la contrarier.

\section{Références}

Billet, Ph., 2005. L'évaluation des incidences des documents d'urbanisme sur l'environnement. JCP AdministrationsCollectivités territoriales, act. $\mathrm{n}^{\circ} 270$.

Blandin P., Luce J.-M., Vignon V., 1999. L'impact de l'autoroute A28 sur les populations sarthoises de trois espèces de coléoptères protégées au titre de la Directive "Habitats » (Osmoderma eremita, Lucanus cervus et Cerambyx cerdo). Diagnostic et préconisations, Rapport final. Muséum national d'histoire naturelle pour COFIROUTE.

Burdeau, M., Blaise L., Fourbier Y., Taillier M., 2001. L'impact de l'autoroute A28 et de ses opérations connexes sur les habitats et les populations d'Osmoderma eremita, du Mans à Tours. Rapport de l'Inspection générale de l'environnement et du Conseil général du Génie rural, des eaux et forêts, ministère de l'Environnement.

Busson, B., 2001. L'urgence en matière de protection de l'environnement, avancées et limites du référé administratif, $D r$. Env., 88, 93-96.

Chauvin, N., 1996. L'illégalité du plan d'occupation des sols, Litec.

Duguet, R., Melki, F. (Eds), 2003. Les amphibiens de France, Belgique et Luxembourg, Mèze, Éd. Biotope.

Holleaux, A., 1981. La jurisprudence du bilan, PresseEnvironnement, 425, 3-8.

Lesbarrères, D., 2003. Déplacements et mouvements migratoires des anoures : le problème de l'utilisation des «crapauducs", in C. Baudoin (Ed.), L'éthologie appliquée aujourd'hui, Vol. 2, Gestion des espèces et des habitats, Neuilly-sur-Seine, Éditions Ed.

Rouvillois, F., 1997, note sous Cons. d'État, Assemblée, 28 mars 1997, Association contre le projet de l'autoroute transchablaisienne, Revue française de droit administratif, 748-753.

Service d'études techniques des routes et autoroutes (SETRA), 1985. Routes et faune sauvage, Actes du colloque de Strasbourg, Conseil de l'Europe, 5-7 juin 1985, ministère de l'Équipement.

Service d'études techniques des routes et autoroutes (SETRA), 1993. Passages pour la grande faune. Guide technique, SETRA.

Service d'études techniques des routes et autoroutes (SETRA), 2000. Fragmentation de l'habitat due aux infrastructures de transport - État de l'art, ministère de l'Équipement.

Untermaier, J., 1986. De la compensation comme principe général du droit et de l'implantation des télésièges en site classé, note sous CE, 27 novembre 1985, Commune de ChamonixMont-Blanc, Revue juridique de l'environnement, 381-412.

Vallauri, D., André, J., Blondel, J., 2002. Le bois mort, un attribut vital de la biodiversité de la forêt naturelle, une lacune des forêts gérées. Rapport WWF, Paris.

37 Cons. d'État, 22 janvier 2003, Commune de Val d'Isère et Association des montagnards de la Vanoise, JCP AdministrationsCollectivités territoriales 2003, n 1561, note Ph. Billet; Environnement, août-septembre 2003, p. 24, note L. Benoît.

38 JO 2 juin 2005, p. 9886.

39 v. Trib. adm. Pau, 19 décembre 2002, SEPANSO Béarn Pyrénées, préc. note 19, à propos de l'aménagement d'un site d'accueil pour les espèces concernées et leur déplacement vers ce site. Ainsi que Cons. d'État, 14 novembre 2003, Assoc. Robin des Bois et autres : JCP Administrations-Collectivités territoriales 2004, $\mathrm{n}^{\circ} 1263$, note Ph. Billet.

Voir également l'arrêté préfectoral du 5 juillet 2004 du préfet de l'Orne d'autorisation de capture temporaire d'espèces d'Osmoderma eremita Pique prune à des fins scientifiques ou de sauvegarde liées au projet d'axe autoroutier A88 Caen-Alençon, dans sa portion traversant le bocage ornais. Celui-ci autorise ainsi : «1. Prélèvement, transport et restitution dans des cavités d'arbres favorables de terreaux abritant le Pique-prune récolté sur les arbres identifiés sur l'emprise du chantier de l'infrastructure en projet. L'ordre des couches prélevées dans la cavité initiale sera respecté lors du dépôt dans la cavité d'accueil. 2. Prélèvement transport de fûts à cavité abritant potentiellement le Pique-prune, pour les transférer sur des sites d'accueil favorables ». 